\title{
Wavelet-based EVM Testing for Non-cooperative Communication Signals
}

\author{
Min Han ${ }^{1}$, Zhijin $\mathrm{Su}^{1}$, Honglin Wan ${ }^{2}$, Jianhua $\mathrm{Xu}^{3}$ \\ ${ }^{1}$ School of Information Science and Engineering Shandong University, Shandong Province 250100, China \\ ${ }^{2}$ School of Physics and Electronic Science Shandong Normal University, Shandong Province, China \\ ${ }^{3}$ The $41^{\text {st }}$ Institute of China Electronics Technology Group Corporation, Shandong Province, China \\ hanmin@sdu.edu.cn suzhijin@163.com visage1979@sdu.edu.cn xujianhua@ei41.com
}

\begin{abstract}
The error vector magnitude (EVM) is extensively applied as a metric for digital transmitter signal quality compliance in modern communication systems. In cooperative communication, signal carrier frequency and symbol rate are predicted, and the reference signal can be recovered from demodulated symbols directly. However in non-cooperative communications, the signal carrier frequency and symbol rate are estimated. Since the demodulation and EVM are very sensitive to symbol rate, we focus on the EVM of non-cooperative communication in this paper. In our proposal, a novel wavelet-based EVM recovery of reference signal is presented. This method can recover reference signal accurately to achieve the true EVM, if the estimation error of symbol rate has been bounded within a small range. The experimental results depict the validity of our algorithm.

Index Terms - EVM, Wavelet, Non-cooperative communication.
\end{abstract}

\section{Introduction}

The error vector magnitude (EVM) is extensively applied as a metric for digital transmitter signal quality compliance in modern communication systems, that is used to measure the difference between the expected complex voltage value of a demodulated symbol and the value of the actual received symbol. Moreover, the bit error rate gives a "go," "no-go" level of system characterization. EVM can be more useful to the microwave engineering because it contains information about both amplitude and phase errors of the signal, [1], [2]. EVM encapsulates many nonidealities of the transmitter, including inter symbol interference, mismatches, non-linearity, phase noise and spurs, and carrier leakage [3]. Such additional information allows a more complete picture of the channel distortion and is more closely related to the physics of the system.

The predecessors have accumulated a lot of research findings [4], [5], [6] of EVM measurement algorithms for cooperative communication systems. However, these methods cannot be adopted to non-cooperative communication systems. In cooperative communication systems, there is much prior knowledge on receivers and signal, many data-aided algorithms may be adopted in the receiver. However in noncooperative communication systems, there is almost no knowledge on the intercepted signal of receiver. In this case, only blind signal processing can be used. How to capture information from a received signal without any prior knowledge is still an important and difficult problem to be solved.

To calculate the EVM in non-cooperative communication, the parameters of the signal must be estimated first, and the accuracy of these parameters estimated will affect EVM precision greatly. For example, small errors of the frequency offset or the symbol rate estimated will lead to a large error of EVM estimated. In this paper, a novel EVM calculation method based on wavelet method is proposed. In our algorithm, if symbol rate estimation error is within a certain range, the influence of the parameters estimated on the EVM can be effectively restricted. In this paper, Haar wavelet is used to locate the transition point and recover the reference signal.

\section{Error Vector Magnitude}

In wireless communication systems, the magnitude and phase of signals in receiver are different from that in transmitters due to nonideal modulation and transmission. From another point of view, measured vectors are different from ideal vectors (or reference vectors) in the complex plane and error vectors are used to represent the difference. Figure 1 illustrates the concepts of measured vectors, reference vectors and error vectors. EVM defines the relationship between error vectors and reference vectors which is given by:

$$
E V M_{\text {RUS }}=\sqrt{\frac{\sum_{k=0}^{N-1}|e(k)|^{2}}{\sum_{k=0}^{N-1}|s(k)|^{2}}}
$$

Let $e(k)=s(k)-z(k)$, Where $s(k)$ is the reference sequence of complex symbols, $z(k)$ is the measured sequence of complex symbols, after best-fit procedure, and $e(k)$ is the complex error sequence. The symbol error vector of $e(k)$ is formed using the best-fit amplitude normalized, time synchronized, and frequency/phase aligned sampled symbol waveform over the measurement time interval. In practical measurement of EVM, if the explicit reference waveform is not available, demod-remod is used to generate a reference waveform.

\footnotetext{
* This work is partially supported by National Foundation of China (Key Program) \#9140A170301 to Jianhua Xu.
} 


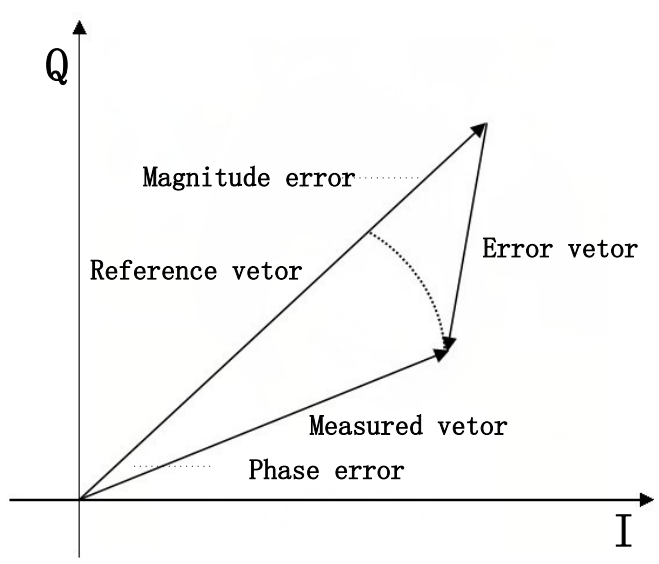

Fig 1. Error Vector Magnitude

\section{EVM testing flow}

As shown in Figure 2, in a cooperative communication, the symbol rate used in the ideal modulation process is known exactly in advance, and reference signal can be directly obtained by modulating[7], [8]. However, in a non-cooperative communication, as shown in Figure 3, carrier frequency, symbol rate estimated information, and the estimation accuracy of these parameters, affect that of the EVM. Specifically, the error of offset estimation will lead to phase drift of baseband signal, and the symbol rate error will result in that the reference signal and the measuring signal cannot be synchronized, as shown in Figure 4. Even if the signal is the same as the initial phase, and the time domain synchronization, the accumulated error will corrupt the synchronization of the reference signal and the measuring signal.

Considering this issue, in this paper we propose a novel method of calculation of EVM. The wavelet-based approach detects the symbol transition point and recover the reference signal. If the estimation of symbol rate has a bounded bias, our proposal can also provide a reasonable reference signal to eliminate the influence of inaccurate estimation of the signal.

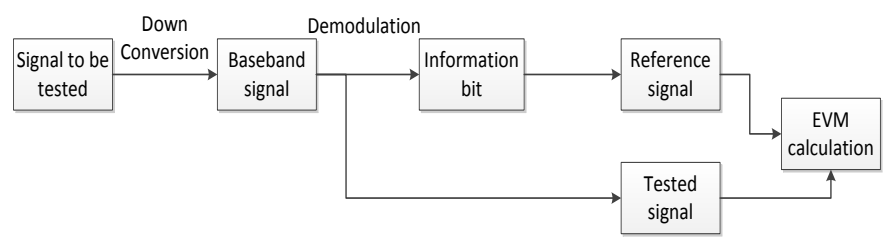

Fig 2. EVM calculation process of cooperative communication baseband signal

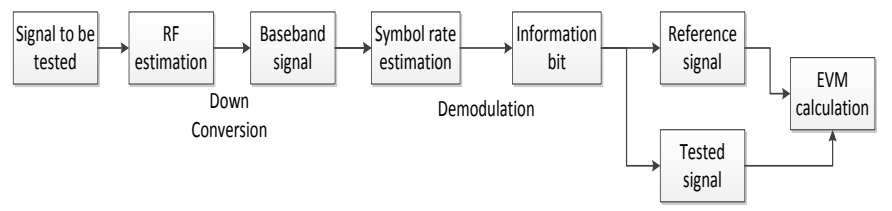

Fig3. EVM calculation process of non-cooperative communication baseband signal

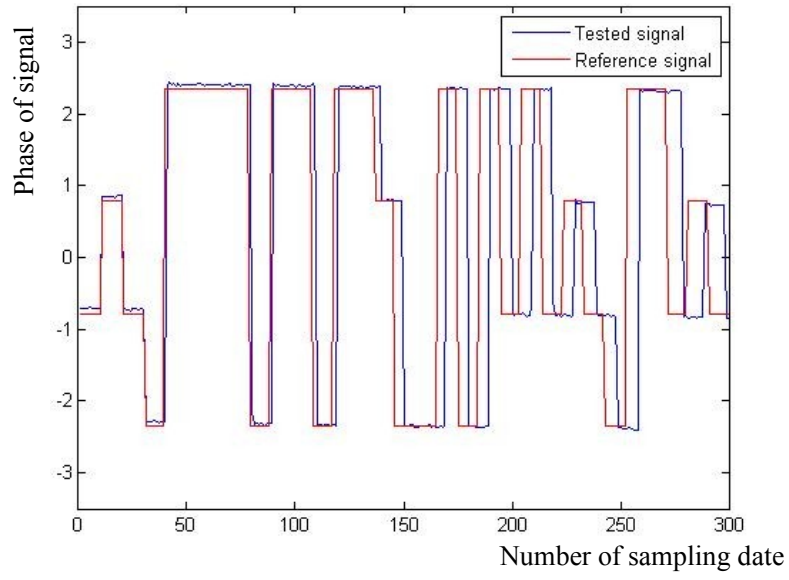

Fig 4. The comparison between the reference signal directly recovered and the measured signal

\section{A. Wavelet Model}

A communication signal analytical expression is

$$
x(t)=s(t)+n(t)=\tilde{s}(t) e^{j\left(\omega_{c} t+\theta_{c}\right)}+n(t)
$$

Wherein, $x(t)$ is a complex signal, $s(t)$ is the modulated complex signal, $n(t)$ is the additive white Gaussian noise, $\omega_{c}$ is carrier angular frequency, $\theta_{c}$ is a carrier initial phase, and $\tilde{s}(t)$ is the base band signal. The MPSK signals, for example, can be expressed as:

$$
\tilde{s}(t)=A \sum_{n} e^{j \phi_{n}} u\left(t-n T_{s}\right)
$$

Where $A$ is the signal amplitude, $\phi_{n}$ is the phase of the n-th element of the signal, $u(t)$ is a unit step function, $T_{s}$ is a symbol length.

In this paper, using continuous wavelet Transform[9],[10]:

$$
C W T(a, \tau)=\frac{1}{\sqrt{a}} \int s(t) \psi^{*}\left(\frac{t-\tau}{a}\right) d t
$$

Where $s(t)$ is the signal being measured, $\psi(t)$ is the mother wavelet function, * represents a conjugate. $a$ is the scale, and $\tau$ is the displacement.

We chose Haar wavelet and its expression with the follows[11] :

$$
\psi(t)= \begin{cases}1, & -0.5<t<0 \\ -1, & 0<t<-0.5 \\ 0, & \text { others }\end{cases}
$$

Wavelet transform of digital MPSK signal is divided into the phase continuous interval and phase discontinuity interval. In phase continuous interval:

$$
|C W T(a, \tau)|=\frac{A}{\sqrt{a}} \frac{4}{\omega_{c}} \sin ^{2} \frac{a}{4} \omega_{c}
$$


In the discontinuous phase interval, $\varphi_{n}, \varphi_{n+1}$, respectively, for the phase of the signal of the $n$-th and $(\mathrm{n}+1)$-th symbols, and at $d,(d<0)$ :

$$
\begin{aligned}
& |C W T(a, \tau)|=\frac{A}{\sqrt{a} \omega_{c}} \mid\left(e^{j \omega_{c} d}-e^{-j \omega_{c} \frac{a}{2}}\right)+ \\
& e^{j\left(\varphi_{n+1}-\varphi_{n}\right)}\left(2-e^{j \omega_{c} d}-e^{j \omega_{c} \frac{a}{2}}\right) \mid
\end{aligned}
$$

Similar results when $d>0$.

From the above two equations, within a cycle or a cycle within the same symbol, the modulus of the wavelet coefficient is a constant. When the symbol transitions, the coefficients will be changed, and in the transition point, a maximum is generated.

\section{B. Wavelet scale selection and the extraction of the extreme points}

The key of extracting symbol transition point is selecting the correct wavelet scale.[12] The smaller the choice of wavelet scales, the higher the accuracy estimated, unfortunately, but more easily influenced by the highfrequency noise, stability correspondingly deteriorated. The larger the choice of wavelet scales, the better stability estimated, however, when scale beyond the length of the symbol, the symbol transitions can not be detected. Taking into account the cyclical nature of the signal sign change, select $a=c / R,(0.4<c<1)$ as wavelet transform scale [13], wherein $R$ is the estimated value of the signal symbol rate[14]. This can effectively resist the influence of high frequency noise, to ensure the detection of symbol transition.

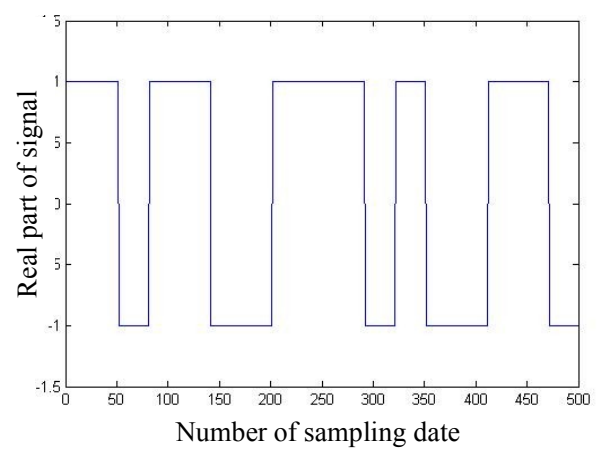

(a)

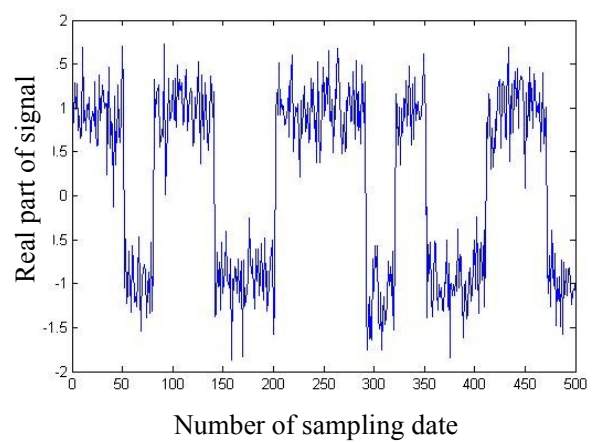

(b)

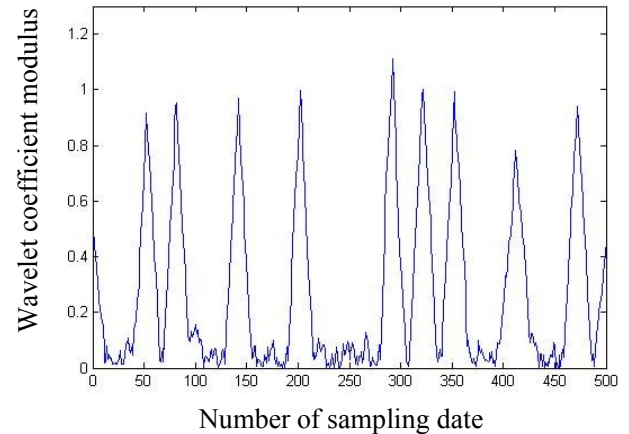

(c)

Fig 5. signal waveform and the wavelet transform (a)BPSK modulated baseband signal without noise. (b)BPSK modulated baseband signal with noise. (c)Wavelet transform coefficient modulus value

As can be seen from Figure 5, the wavelet coefficient modulus maxima correspond to the symbolic transition point, consistent with the theoretical analysis of the results. In order to eliminate the the noise pseudo extreme points caused by envelope fluctuations, and extract modulus maxima [14], we select a threshold value threshold $T_{h}$ to denoise $|C W T(a, \tau)|$.For BPSK signals, we select $T_{h}=\max (|C W T(a, \tau)|) / 2$ (large number of simulation experiments permit this threshold for BPSK, QPSK, and 8PSK signal have good results in different SNR) as the denoising door limit threshold. The wavelet coefficients modulus value of the signal after denoising can be expressed as:

$$
|C W T \tilde{(}(a, \tau)|=\left\{\begin{array}{cl}
|C W T(a, \tau)| & |C W T(a, \tau)| \geq T_{h} \\
0 & |C W T(a, \tau)|<T_{h}
\end{array}\right.
$$

Set $0.5 / \hat{R}$ as window length, extract local maxima of $|C W T \tilde{(}(a, \tau)|$ to locate the symbol transition point $P_{i},(1<i<N)$, where $i$ represents the serial number of the transition point, and $N$ is the number of transition points.

Set the extreme point as symbol switching point, set judgment of the mean phase between the two transition points as the phase value between switching point, set the mean amplitude of the signal as amplitude, we can get the reference signal. This signal as the reference signal can effectively eliminate the reference signal recovery errors caused by symbol rate estimation deviation.

\section{Simulation and analysis}

In order to evaluate the EVM measurement algorithm proposed in this paper, a simulation platform is set up. The signal is generated by signal generator and processed by Matlab software. We employ QPSK and BPSK as measuring signal modulation scheme, with the signal sampling rate $F S=30 \mathrm{M}$, the symbol rate $R=1 \mathrm{M}, S N R=20 \mathrm{~dB}$, the estimation error of the symbol rate is $5 \%$. Figure 6 (a) is the comparison of the reference signal recovered directly by the symbol rate estimated and the measured signal. It can be seen, the both signals on the phase of the initial moment is aligned. With the 
time, the phase deviation between reference signal and the measurement signal in the time domain is on the grows, which is the cause of the deviation of the symbol rate estimates. In Figure 6 (b) we use the method in this paper to recover the reference signal. It can be seen that the phase of the reference signal and the measurement signal in the time domain is always consistent. Figure 7 shows the EVM comparison of the proposed method and the direct recovery method with different modulation and signal-to-noise ratio. Also the proposed method has better results under different signal-tonoise ratio and modulation.

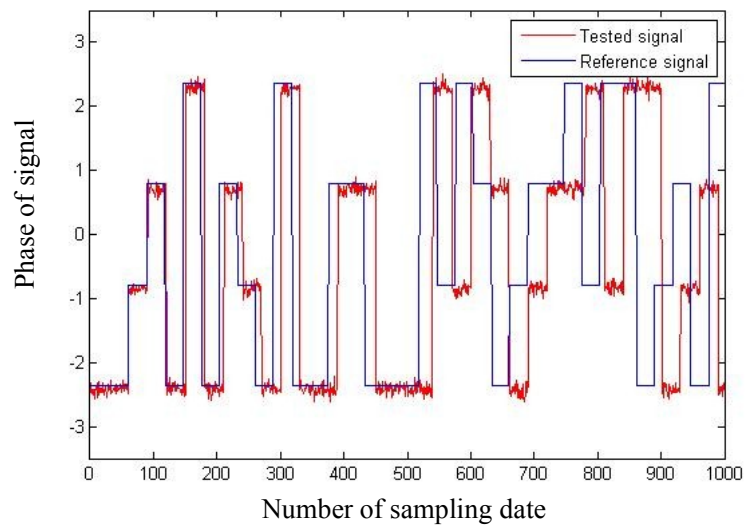

(a)

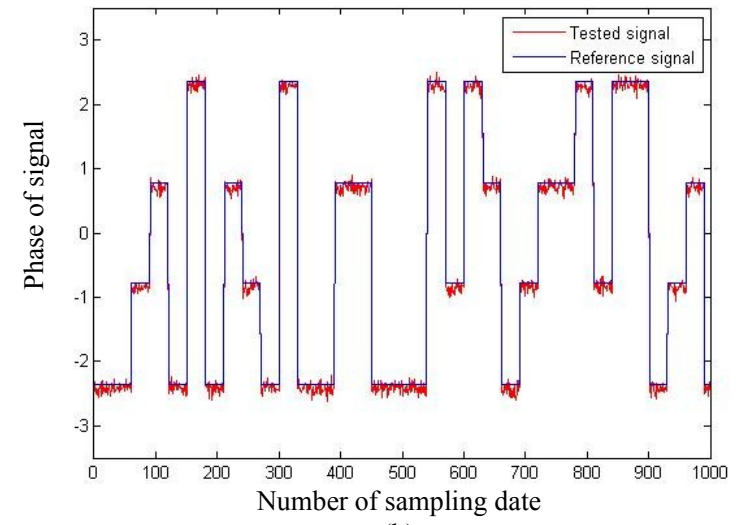

(b)

Fig 6. The comparison between the reference signal and the measured signal(a) Directly recover method(b) Method proposed

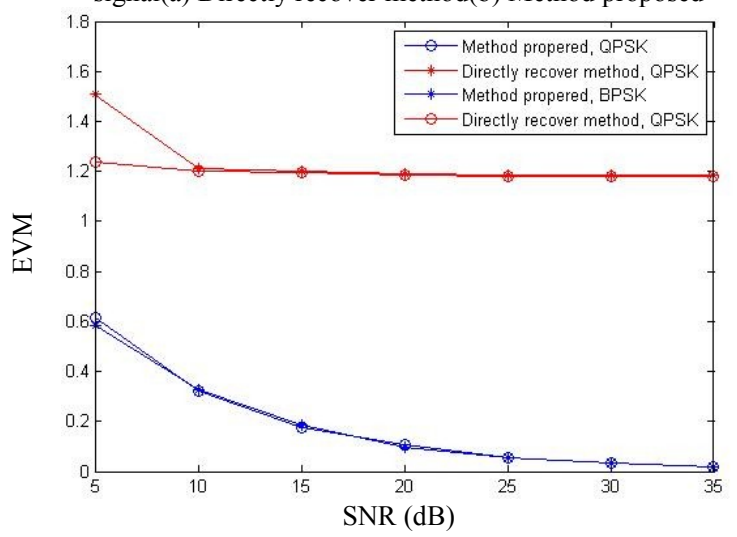

Fig 7. The comparison of EVM between proposed method and directly recover method

\section{Conclusion}

This paper proposes an EVM testing method of noncooperative communication signals based on wavelet detection. This method can recover the reference signal in the case of deviation of estimated symbol rate, and achieve an reasonable EVM calculation. Experimental results show that this method corrects the detection caused by the error parameter estimation, is more robust to noise, and applies EVM calculation to non-cooperative communication signals.

\section{References}

[1] Hassun, Roland ; Flaherty, Michael ; Matreci, Robert J. ;Taylor, Mitch. "Effective evaluation of link quality using error vector magnitude techniques," Proceedings of 1997 Wireless Communications Conference (WCC'97), pp: 89-94 USA. Piscataway, Aug 11-13, 1997.

[2] Wang A K, Ligmanowski R, Castro J, Anthony Mazzara. "EVM simulation and analysis techniques." Proceedings of IEEE Military Communications Conference (Milcom'06), pp: 1-7 USA. Washington, DC, Oct 23-25, 2006.

[3] Q. Gu, "RF System Design of Transceivers for Wireless Communications". Springer Science, 2005.

[4] A. Springer, T. Frauscher, B. Adler, D. Pimingsdorfer R. Weigel. "Impact of nonlinear amplifiers on the UMTS system." IEEE 6th Int. Sypm. on Spreas-Spectrum Tech. \& Appli. vol.2, pp: 465-469 New Jersey,USA, 2000 ,

[5] Ashkan Mashhour, Assaad Borjak. "A method for computing error vector magnitude in GSM EDGE systems-simulation results." IEEE communications letters,vol. 5 ,no. 3, pp.88-91, March 2001.

[6] Michael D. McKinley, et al, "EVM Calculation for Broadband Modulated Signals," In 64th Automatic RF Techniques Group Conference(ARFTG) Conf. Dig., pp. 45-52, Orlando, Dec 2004.

[7] K. Voelker, "Apply error vector measurements in communications design," Microwaves \& RF, vol. 34, pp. 143-152, 1995.

[8] R.Williams and J. Getchell, "High-speed measurement of digital wireless system SNR by means of error vector magnitude analysis," in Proc. Wirel. Commun. Conf., Boulder, CO, 1996, pp. 68-70.

[9] I. Daubechies, "Ten Lectures on Wavelets, Philadelphia," PA: Society for industrial and applied mathematics, 1992.

[10] M. Holschneider, et al, Wavelets: An Analysis Tool, Physics Today, vol. 49, no. 28, pp. 65, August 1996.

[11] Haar A. Zur theorie der orthogonalen Funktionsysteme. Mathematische Annalen, vol.69, no.3, pp. 331-371, 1910.

[12] Su Zhijin; Xu Jianhua and Han Min. "Symbol rate estimation of MPSK short signals based on wavelet transform," Journal of electronic measurement and instrument, vol.35, no. 2, pp, 140-144, February 2013

[13] Deng Zhenmiao, Liu Yu, "Phase-domain blind estimation of symbol duration based on Haar wavelet transform," Journal of Systerms Engineering and Electronics, vol. 21, no.3, pp. 375-381, 2010.

[14] Sun Gangcan, An Jianping, Yang Jie, Yang Jing, Li Zhijun, "Symbol Rate Estimation Using Cyclic Correlation and Haar Wavelet Transform, Wireless Communications," Networking and Mobile Computing, WiCOM'08. 4th International Conference on. IEEE, pp. 1021-1024, New York, 12-14 Oct. 2008 